LINEAR AND NON-LINEAR THEORY OF

GENERALIZED FUNCTIONS AND ITS APPLICATIONS

BANACH CENTER PUBLICATIONS, VOLUME 88

INSTITUTE OF MATHEMATICS

POLISH ACADEMY OF SCIENCES

WARSZAWA 2010

\title{
RECENT PROGRESS IN SPECIAL COLOMBEAU ALGEBRAS: GEOMETRY, TOPOLOGY, AND ALGEBRA
}

\author{
M. KUNZINGER \\ Faculty of Mathematics, University of Vienna \\ Nordbergstraße 15, A-1090 Wien, Austria \\ E-mail: michael.kunzinger@univie.ac.at
}

\begin{abstract}
Over the past few years there has been considerable progress in the structural understanding of special Colombeau algebras. We present some of the main trends in this development: non-smooth differential geometry, locally convex theory of modules over the ring of generalized numbers, and algebraic aspects of Colombeau theory. Some open problems are given and directions of further research are outlined.
\end{abstract}

1. Introduction. Colombeau algebras of generalized functions [4, 5, 23, are differential algebras that contain the vector space of Schwartz distributions as a linear subspace, and the space of smooth functions as a faithful subalgebra. Initially discovered in the context of infinite-dimensional calculus in locally convex spaces, such algebras have turned out to be a powerful tool in the study of singular problems that involve differentiation combined with non-linear operations. In particular, Colombeau algebras quickly found (and continue to find) applications in the field of non-linear partial differential equations (e.g., [23, 3, 22]), where the application of classical distributional methods is limited by the impossibility to consistently define an intrinsic product of distributions [27.

From the mid 1990's, it also became apparent that Colombeau algebras could be a significant tool with which to study singular problems in various geometrical settings. In particular, early work centered on applications to problems in General Relativity (see 28] for a recent survey), and Lie group analysis of partial differential equations (e.g., [16, 14]).

2000 Mathematics Subject Classification: Primary 46F30; Secondary 46T30, 46A20, 16D25, 53B20.

Key words and phrases: special Colombeau algebras, non-smooth differential geometry, locally convex modules, ring of generalized numbers.

The paper is in final form and no version of it will be published elsewhere. 
At the same time, structural properties of Colombeau algebras came to the fore in the work of several research groups. In particular, a thorough study of algebraic properties was carried out (e.g., 11, 29]) and topological and functional analytic structures on Colombeau spaces were developed and refined to a high degree (e.g., [25, 26, 9, 10]).

The aim of this contribution is to provide an overview of some of these developments that show significant potential both for the intrinsic understanding of algebras of generalized functions and for applications in geometry, differential equations, and mathematical physics.

2. Non-smooth differential geometry. Throughout this paper we will employ the so-called special (or simplified) version of Colombeau's algebras. To fix notations we briefly recall the definition of the Colombeau algebra $\mathcal{G}(M)$ on a manifold $M$ (see, e.g., [14]). Let $\mathcal{P}(M)$ denote the space of linear differential operators on $M \cdot \mathcal{G}(M)$ is defined as the quotient space $\mathcal{E}_{m}(M) / \mathcal{N}(M)$, where the spaces of moderate resp. negligible nets are defined by

$$
\begin{aligned}
\mathcal{E}_{m}(M) & =\left\{\left(u_{\varepsilon}\right)_{\varepsilon} \in \mathcal{C}^{\infty}(M)^{(0,1]}: \forall K \subset \subset M \forall P \in \mathcal{P}(M) \exists l \sup _{x \in K}\left|P u_{\varepsilon}(x)\right|=O\left(\varepsilon^{-l}\right)\right\}, \\
\mathcal{N}(M) & =\left\{\left(u_{\varepsilon}\right)_{\varepsilon} \in \mathcal{E}_{m}(M): \forall K \subset \subset M \forall m \sup _{x \in K}\left|u_{\varepsilon}(x)\right|=O\left(\varepsilon^{m}\right)\right\} .
\end{aligned}
$$

Here and in what follows we will assume that all representatives of generalized functions in fact depend smoothly on the regularization parameter $\varepsilon$. A similar definition can be given for the space $\Gamma_{\mathcal{G}}(M, E)$ of generalized sections of a vector bundle $E \rightarrow M$, and we have the fundamental $\mathcal{C}^{\infty}(M)$-module isomorphism

$$
\Gamma_{\mathcal{G}}(M, E) \cong \mathcal{G}(M) \otimes_{\mathcal{C}^{\infty}(M)} \Gamma(M, E),
$$

i.e., generalized sections may be viewed globally as sections with generalized coefficient functions. Based on regularization operations via convolution in charts (cf. the de Rham regularizations in [8]) it can be shown that there exist injective sheaf morphisms

$$
\iota: \Gamma\left(_{-}, E\right) \hookrightarrow \mathcal{D}^{\prime}\left(_{-}, E\right) \hookrightarrow \Gamma_{\mathcal{G}}\left({ }_{-}, E\right) .
$$

An important feature distinguishing Colombeau generalized functions from Schwartz distributions is the availability of a point value characterization: we call a net $\left(x_{\varepsilon}\right)_{\varepsilon}$ of points in $M$ compactly supported if $x_{\varepsilon}$ remains in some compact set for $\varepsilon$ small. Two compactly supported nets $\left(x_{\varepsilon}\right)_{\varepsilon},\left(y_{\varepsilon}\right)_{\varepsilon}$ are called equivalent, $\left(x_{\varepsilon}\right)_{\varepsilon} \sim\left(y_{\varepsilon}\right)_{\varepsilon}$, if $d_{h}\left(x_{\varepsilon}, y_{\varepsilon}\right)=O\left(\varepsilon^{m}\right) \forall m$, where $d_{h}$ is the distance function induced by any Riemannian metric $h$ on $M$. The quotient space $\tilde{M}_{c}:=M^{(0,1]}$ is called the space of compactly supported generalized points. Then we have (cf. [14, Th. 3.2.8):

TheOREM 2.1. Let $u \in \mathcal{G}(M)$. Then $u=0$ if and only if $u(\tilde{x})=0$ for all $\tilde{x} \in \tilde{M}_{c}$.

As a first pointer at algebraic properties of $\mathcal{G}$, let us have a look at the question of (multiplicative) invertibility in both $\mathcal{G}(M)$ and the ring of constants in $\mathcal{G}(M)$ (or space of generalized numbers), $\tilde{\mathbb{K}}$ (for $\mathbb{K}=\mathbb{R}$ resp. $\mathbb{K}=\mathbb{C}$ ).

LEMma 2.2. Let $u \in \mathcal{G}(M)$. The following are equivalent:

(i) $u$ is invertible. 
(ii) $u(\tilde{x})$ is invertible in $\tilde{\mathbb{K}}$ for all $\tilde{x} \in \tilde{M}_{c}$.

(iii) $u$ is strictly nonzero, i.e., $\forall K \subset \subset M \exists q$ s.t. $\inf _{p \in K}\left|u_{\varepsilon}(p)\right|>\varepsilon^{q}$ for $\varepsilon$ small.

Similarly, for generalized numbers we have:

LEMma 2.3. Let $r \in \tilde{\mathbb{K}}$. The following are equivalent:

(i) $r$ is invertible.

(ii) $r$ is not a zero divisor.

(iii) $r$ is strictly nonzero.

(iv) For every representative $\left(r_{\varepsilon}\right)_{\varepsilon}$ of $r$ there exists some $\varepsilon_{0}>0$ such that $r_{\varepsilon} \neq 0$ for all $\varepsilon<\varepsilon_{0}$.

While the other conditions in Lemmas 2.2 and 2.3 are well-known (cf. [14]), (iv) from Lemma 2.3 is a rather recent and very convenient observation from [21].

For applications in general relativity, a notion of generalized (pseudo-)Riemannian metric is of central importance. Denoting $\Gamma_{\mathcal{G}}\left(M, T_{s}^{r} M\right)$ by $\mathcal{G}_{s}^{r}(M)$ we have the following characterization $([17,21])$ :

THEOREM 2.4. Let $g \in \mathcal{G}_{2}^{0}(M)$. The following are equivalent:

(i) $g: \mathcal{G}_{0}^{1}(M) \times \mathcal{G}_{0}^{1}(M) \rightarrow \mathcal{G}(M)$ is symmetric and $\operatorname{det}(g)$ is invertible in $\mathcal{G}(M)$.

(ii) For each chart $(\psi, V), \forall \tilde{x} \in(\psi(V))_{c}^{\sim}: \psi_{*} g(\tilde{x}): \tilde{\mathbb{K}}^{n} \times \tilde{\mathbb{K}}^{n} \rightarrow \tilde{\mathbb{K}}$ is symmetric and nondegenerate.

(iii) $\operatorname{det}(g)$ is invertible in $\mathcal{G}(M)$ and $\forall \bar{V} \subset \subset M$ there exists a representative $\left(g_{\varepsilon}\right)_{\varepsilon}$, such that each $\left.g_{\varepsilon}\right|_{V}$ is a smooth pseudo-Riemannian metric.

Moreover, if $g$ satisfies these equivalent conditions then $g$ has index $j$ if and only if for each chart $\psi$ and each $\tilde{x}, \psi_{*} g(\tilde{x})$ is a symmetric bilinear form on $\tilde{\mathbb{R}}^{n}$ with index $j$.

As in the smooth setting, the following fundamental lemma shows that each generalized pseudo-Riemannian metric induces a unique Levi-Civita connection ([17]):

THEOREM 2.5. For any generalized pseudo-Riemannian metric $g$ on $M$ there exists a unique connection $\hat{\nabla}: \mathcal{G}_{0}^{1}(M) \times \mathcal{G}_{0}^{1}(M) \rightarrow \mathcal{G}_{0}^{1}(M)$ such that:

$(\nabla 1) \hat{\nabla}_{X} Y$ is $\tilde{\mathbb{R}}$-linear in $Y$.

$(\nabla 2) \hat{\nabla}_{X} Y$ is $\mathcal{G}(M)$-linear in $X$.

$(\nabla 3) \hat{\nabla}_{X}(u Y)=u \hat{\nabla}_{X} Y+X(u) Y$ for all $u \in \mathcal{G}(M)$.

$(\nabla 4)[X, Y]=\hat{\nabla}_{X} Y-\hat{\nabla}_{Y} X$

$(\nabla 5) X\langle Y, Z\rangle=\left\langle\hat{\nabla}_{X} Y, Z\right\rangle+\left\langle Y, \hat{\nabla}_{X} Z\right\rangle$

With these tools at hand, one can proceed to analyzing curvature quantities and geodesics for singular metrics. We refer to [28] for a recent overview of applications in general relativity. More generally, generalized connections in principal fiber bundles have been studied in [19. Notions like curvature, holonomy and characteristic classes can then be modelled in a non-smooth setting. First applications to singular Yang-Mills equations can also be found in [19].

A further aspect of Colombeau algebras that allows one to go beyond the distributional setting is the notion of generalized functions taking values in differentiable manifolds ([15, 18]). The basic idea is to consider, for given manifolds $M, N$, a quotient construction 
on subspaces of $\mathcal{E}[M, N]:=\mathcal{C}^{\infty}(M, N)^{(0,1]}$. The corresponding growth conditions can either be modelled by asymptotic estimates in charts (15]) or, more elegantly, using 'referee functions' for testing for moderateness resp. negligibility, as follows: we say that a net $\left(u_{\varepsilon}\right)_{\varepsilon} \in \mathcal{E}[M, N]$ (depending smoothly on $\varepsilon$ ) is c-bounded if for each $K \subset \subset M$ there exists some $K^{\prime} \subset \subset N$ and some $\varepsilon_{0}$ such that $u_{\varepsilon}(K) \subseteq K^{\prime}$ for all $\varepsilon<\varepsilon_{0}$. A c-bounded net $\left(u_{\varepsilon}\right)_{\varepsilon}$ is called moderate if $\left(f \circ u_{\varepsilon}\right)_{\varepsilon} \in \mathcal{E}_{m}(M) \forall f \in \mathcal{C}^{\infty}(N)$. The space of moderate nets is denoted by $\mathcal{E}_{m}[M, N]$. Two elements $\left(u_{\varepsilon}\right)_{\varepsilon},\left(v_{\varepsilon}\right)_{\varepsilon}$ of $\mathcal{E}_{m}[M, N]$ are called equivalent, $\left(u_{\varepsilon}\right)_{\varepsilon} \sim\left(v_{\varepsilon}\right)_{\varepsilon}$, if $\left(f \circ u_{\varepsilon}-f \circ v_{\varepsilon}\right)_{\varepsilon} \in \mathcal{N}(M) \forall f \in \mathcal{C}^{\infty}(N)$. The space of Colombeau generalized functions on $M$ taking values in $N$ is then given by $\mathcal{G}[M, N]:=\mathcal{E}_{m}[M, N] / \sim$.

Manifold-valued generalized functions are a necessary prerequisite for addressing problems like determining geodesics of singular metrics or flows of generalized vector fields. Based on $\mathcal{G}[M, N]$, a functorial theory of manifold-valued generalized functions and generalized vector bundle homomorphism has been developed in [15, 18.

On the structural level, a basic question is whether $\mathcal{G}[-, N]$ forms a sheaf. Due to the lack of algebraic structure on the target space $N$, the usual tools like partitions of unity are not directly available to answer this question. Nevertheless, we have:

TheOrem 2.6. $\mathcal{G}[-, N]$ is a sheaf of sets.

Sketch of proof. The nontrivial part is to show that any coherent family of locally defined generalized maps is given as a family of restrictions of one globally defined generalized map. The strategy is to use a Whitney embedding of $N$ into some $\mathbb{R}^{n}$ and then apply a gluing procedure in $\mathbb{R}^{n}$ based on partitions of unity. In order to obtain a global representative taking values in $N$, the retraction map of a tubular neighborhood of $N$ in $\mathbb{R}^{n}$ is employed. For details, see [20].

By a similar method, we obtain the following result on the inclusion of continuous maps in $\mathcal{G}[M, N]\left(\sigma\right.$ denotes the identical embedding $f \mapsto(f)_{\varepsilon}$ of $\mathcal{C}^{\infty}(M, N)$ in $\left.\mathcal{G}[M, N]\right)$, cf. [20]:

THEOREM 2.7. There exists an embedding $\iota: \mathcal{C}(M, N) \hookrightarrow \mathcal{G}[M, N]$ with the following properties:

(i) $\iota$ is a sheaf morphism.

(ii) $\left.\iota\right|_{\mathcal{C} \infty}(M, N)=\sigma$.

(iii) $\iota(u)_{\varepsilon}$ converges to $u$ uniformly on compact sets.

As an added benefit, the construction of $\mathcal{G}[M, N]$ provides a blueprint for defining a space of manifold-valued distributions, as follows: set $\mathcal{A}[M, N]=\{u \in \mathcal{G}[M, N] \mid \forall f \in$ $\left.\mathcal{C}^{\infty}(N), \exists \lim _{\varepsilon \rightarrow 0} f \circ u_{\varepsilon} \in \mathcal{D}^{\prime}\right\}$ and let $u \approx_{\mathcal{M}} v$ if for all $f \in \mathcal{C}^{\infty}(N), f \circ u_{\varepsilon}-f \circ v_{\varepsilon} \rightarrow 0$ in $\mathcal{D}^{\prime}$. Then set $\mathcal{D}^{\prime}(M, N):=\mathcal{A}[M, N] / \approx_{\mathcal{M}}$. For $M, N$ Euclidean spaces, $\mathcal{D}^{\prime}(M, N)$ singles out a subspace of bounded distributions. Further properties (e.g., the relationship to Young measures) are analyzed in [20].

3. Some algebraic aspects of Colombeau algebras on manifolds. Based on the construction in the previous sections, here we will give a few examples indicating the in- 
creasingly important role that an understanding of the algebraic structure of Colombeautype spaces plays in a geometrical context.

To begin with, let us consider the structure of the space of algebra isomorphisms from $\mathcal{G}(M)$ to $\mathcal{G}(N)$. In the smooth setting, it has been known for a long time that for any algebra isomorphism $\phi: \mathcal{C}^{\infty}(M) \rightarrow \mathcal{C}^{\infty}(N)$ there is a unique diffeomorphism $f: N \rightarrow M$ of the underlying manifolds such that $\phi$ is given as the pullback map under $f: \phi=u \mapsto u \circ f$. The analogous problem for isomorphisms of Colombeau algebras has only recently been solved by H. Vernaeve in [30]. The result is based on the solution of 'Milnor's exercise' in the Colombeau setting, i.e. the characterization of multiplicative linear functionals on $\mathcal{G}$ :

THEOREM 3.1. Every multiplicative linear functional on $\mathcal{G}(M)$ is of the form

$$
e \delta_{\tilde{x}}: u \mapsto e u(\tilde{x})
$$

for $\tilde{x}$ a generalized point and $e \in \tilde{\mathbb{K}}$ idempotent.

Using this result, we obtain

THEOREM 3.2. Let $\phi: \mathcal{G}(M) \rightarrow \mathcal{G}(N)$ be an algebra-isomorphism (with $\phi(1)=1)$. Then $\phi=f^{*}$ for some $f \in \mathcal{G}[N, M]$ such that $f^{-1} \in \mathcal{G}[M, N]$. Also, $\phi^{-1}=f_{*}$.

Next, let us investigate generalized de Rham cohomology. We denote by $\Omega_{\mathcal{G}}^{p}(M)=$ $\Gamma_{\mathcal{G}}\left(M, \Lambda^{p}(M)\right)$ the space of generalized $p$-forms on $M$. Also, as in the smooth setting we introduce the cohomology spaces by

$$
\begin{aligned}
Z_{\mathcal{G}}^{p}(M) & :=\left\{\omega \in \Omega_{\mathcal{G}}^{p}(M) \mid d \omega=0\right\}, \\
B_{\mathcal{G}}^{p}(M) & :=\left\{\omega \in \Omega_{\mathcal{G}}^{p}(M) \mid \exists \tau \in \Omega_{\mathcal{G}}^{p-1}: \omega=d \tau\right\}, \\
H_{\mathcal{G}}^{p}(M) & :=Z_{\mathcal{G}}^{p}(M) / H_{\mathcal{G}}^{p}(M) .
\end{aligned}
$$

The relationship between generalized and smooth de Rham cohomology is as follows:

THEOREM 3.3. For any $p \geq 0$ we have the following isomorphism of real vector spaces:

$$
H_{\mathcal{G}}^{p}(M) \cong \tilde{\mathbb{R}} \otimes_{\mathbb{R}} H^{p}(M)
$$

Sketch of proof. Both

$$
0 \longrightarrow \operatorname{ker}(d) \stackrel{d}{\longrightarrow} \Omega_{\mathcal{G}}^{0}(M) \stackrel{d}{\longrightarrow} \Omega_{\mathcal{G}}^{1}(M) \stackrel{d}{\longrightarrow} \ldots
$$

and

$$
0 \longrightarrow \operatorname{ker}(d) \stackrel{i d \otimes d}{\longrightarrow} \tilde{\mathbb{R}} \otimes_{\mathbb{R}} C^{\infty}(M, \mathbb{R}) \stackrel{i d \otimes d}{\longrightarrow} \tilde{\mathbb{R}} \otimes_{\mathbb{R}} \Omega^{1}(M) \stackrel{i d \otimes d}{\longrightarrow} \ldots
$$

are fine resolutions of the sheaf of locally constant Colombeau generalized functions. The result therefore follows from the abstract de Rham theorem. For details, see [19].

This means that the structural difference between generalized and smooth de Rham cohomology is encoded precisely in the algebraic structure of the ring of generalized numbers.

Finally, let us return to the algebraic foundations of pseudo-Riemannian geometry in the Colombeau setting. As can be seen from Th. 2.4 the study of bilinear forms on $\tilde{\mathbb{R}}^{n}$ is of central importance here. We have ([21]):

THEOREM 3.4. Let $v \in \tilde{\mathbb{R}}^{n}$. The following are equivalent: 
(i) For any positive definite bilinear form $h, h(v, v)>0$.

(ii) $v$ is free (i.e., for any $\lambda \in \tilde{\mathbb{R}}, \lambda v=0 \Rightarrow v=0$ ).

(iii) $v$ can be extended to a basis of $\tilde{\mathbb{R}}^{n}$.

(iv) For each representative $\left(v_{\varepsilon}\right)_{\varepsilon}$ there exists some $\varepsilon_{0}$ such that for all $\varepsilon<\varepsilon_{0}, v_{\varepsilon} \neq 0$.

Based on this result, causality notions (time-like, space-like, and null vectors) can be introduced and analyzed in the generalized setting. Applications include energy methods for solving wave equations on singular space-times (cf. [13]).

4. Algebraic properties of $\tilde{\mathbb{K}}$. In this section we give a brief overview of known results on the algebraic structure of the ring of generalized numbers. For details and proofs we refer to the original sources [1, 2, 29]. In what follows, topological properties always refer to the sharp topology on $\tilde{\mathbb{K}}$ (cf. the following section).

- $\tilde{\mathbb{K}}$ is a reduced ring, i.e., there are no nontrivial nilpotent elements.

- Elements of $\tilde{\mathbb{K}}$ are either invertible or zero-divisors (cf. Lemma 2.3.

- $e \in \tilde{\mathbb{K}}$ is idempotent $\left(e^{2}=1\right)$ iff $e=e_{S}$, the characteristic function of some $S \subseteq(0,1]$.

- $\tilde{\mathbb{K}}$ possesses uncountably many maximal ideals.

- $\tilde{\mathbb{K}}$ is a complete topological ring.

- The closure of any prime ideal is maximal. Conversely, every maximal ideal is closed.

- Let $I$ be an ideal in $\tilde{\mathbb{K}}$. Then the closure of $I$ is the intersection of all maximal ideals containing $I$.

- $\tilde{\mathbb{K}}$ is not:

- Artinian

- Noetherian

- von Neumann regular

- Every ideal $I$ in $\tilde{\mathbb{K}}$ is convex $(x \in I,|y| \leq|x| \Rightarrow y \in I)$.

- An ideal $I$ is prime iff it is pseudoprime and radical, i.e.:

$-\forall S \subset(0,1]: e_{S} \in I$ or $e_{S^{c}} \in I$, and

$-\forall x \in I: \sqrt{|x|} \in I$.

We note that many of the corresponding properties for $\mathcal{G}$ instead of $\tilde{\mathbb{K}}$ are the subject of ongoing research. We conclude this section with the following interesting connection to the nonstandard space of asymptotic numbers (cf. 24]), established in [29, Th. 7.2:

TheOrem 4.1. Let I be a maximal ideal in $\tilde{\mathbb{K}}$. Let $\mathcal{U}:=\left\{S \subseteq(0,1] \mid e_{S^{c}} \in I\right\}$. Let ${ }^{*} \mathbb{K}$ be the nonstandard field constructed by the ultrafilter $\mathcal{U}$ and let $\rho$ be the infinitesimal with representative $(\varepsilon)_{\varepsilon}$. Then ${ }^{\rho} \mathbb{K}$ is canonically isomorphic to $\tilde{\mathbb{K}} / I$.

5. Topology and functional analysis. Topologies on spaces of Colombeau generalized functions and generalized numbers were originally introduced by D. Scarpalézos by the name of sharp topologies in 1993 (and published only later in [25, 26]). After the field lay dormant for some years (in which the main focus of research was on applications in PDEs) there occurred a veritable surge of activities lately. In particular, the fundamental 
work by C. Garetto [9, 10, has led to the development of a full-scale locally convex theory for algebras of generalized functions. In this section we outline some of the main features of this theory.

For any given locally convex vector space $E$ whose topology is induced by the family of seminorms $\left(p_{i}\right)_{i \in I}$, we set

$$
\begin{aligned}
\mathcal{M}_{E} & :=\left\{\left(u_{\varepsilon}\right)_{\varepsilon} \in E^{(0,1]} \mid \forall i \exists N: p_{i}\left(u_{\varepsilon}\right)=O\left(\varepsilon^{-N}\right)\right\}, \\
\mathcal{N}_{E} & :=\left\{\left(u_{\varepsilon}\right)_{\varepsilon} \in E^{(0,1]} \mid \forall i \forall q: p_{i}\left(u_{\varepsilon}\right)=O\left(\varepsilon^{q}\right)\right\}, \\
\mathcal{G}_{E} & :=\mathcal{M}_{E} / \mathcal{N}_{E} .
\end{aligned}
$$

Then $\mathcal{G}_{E}$ is a $\tilde{\mathbb{C}}$-module. The special Colombeau algebra $\mathcal{G}(\Omega)$ is obtained as the special case $E=\mathcal{C}^{\infty}(\Omega)$ of this construction (cf. [9, 7]).

On $\mathcal{G}_{E}$ we introduce valuations given by

$$
v_{p_{i}}(u):=\sup \left\{b \in \mathbb{R} \mid p_{i}\left(u_{\varepsilon}\right)=O\left(\varepsilon^{b}\right)\right\}
$$

(here $\left(u_{\varepsilon}\right)_{\varepsilon}$ is any representative of $\left.u \in \mathcal{G}_{E}\right)$. The valuations, in turn, induce ultra-pseudoseminorms (ups) via

$$
\mathcal{P}_{i}:=e^{-v_{p_{i}}} .
$$

This family of ups defines the sharp topology on $\mathcal{G}_{E}$. As an important special case we may take $E=\mathbb{C}$, in which case $\mathcal{G}_{E}=\tilde{\mathbb{C}}$. Here we only have one seminorm, $p(x)=|x|$, which induces a valuation $v$ and a corresponding ups denoted by ||$_{e}$.

More generally we may introduce suitable notions for directly generalizing locally convex topologies to the $\tilde{\mathbb{C}}$-module setting. Recall that for $V$ a vector space and $X \subseteq V, X$ is called absorbent in $V$ if $\forall u \in V \exists \lambda_{0} \forall \lambda \geq \lambda_{0}: u \in \lambda X$. Let now $\mathcal{G}$ be a $\widetilde{\mathbb{C}}$-module and let $A \subseteq \mathcal{G}$. If we let $\lambda_{0}$ correspond to the infinitesimal $\left[\left(\varepsilon^{a}\right)_{\varepsilon}\right]$, then $\lambda_{0} \cong\left[\left(\varepsilon^{a}\right)_{\varepsilon}\right] \leq\left[\left(\varepsilon^{b}\right)_{\varepsilon}\right] \cong \lambda$ iff $b \leq a$, so we are led to defining: $A$ is called $\tilde{\mathbb{C}}$-absorbent if $\forall u \in \mathcal{G} \exists a \in \mathbb{R} \forall b \leq a$ : $u \in\left[\left(\varepsilon^{b}\right)_{\varepsilon}\right] A$. Similarly, we call $A \tilde{\mathbb{C}}$-balanced if $\forall \lambda \in \tilde{\mathbb{C}}$ with $|\lambda|_{e} \leq 1: \lambda A \subseteq A$.

To introduce a suitable notion of convexity, recall that a subset $X$ of a vector space $V$ is a convex cone in $V$ if $X+X \subseteq X$ and $\forall \lambda \in(0,1]: \lambda X \subseteq X$. Thus we call a subset $A$ of a $\tilde{\mathbb{C}}$-module $\mathcal{G} \tilde{\mathbb{C}}$-convex if $A+A \subseteq A$ and $\forall b \geq 0$ : $\left[\left(\varepsilon^{b}\right)_{\varepsilon}\right] A \subseteq A$. Finally, we define a locally convex topological $\tilde{\mathbb{C}}$-module to be a topological $\tilde{\mathbb{C}}$-module (which means that + and $\lambda$. are continuous) with a base of $\tilde{\mathbb{C}}$-convex neighborhoods of 0 .

This provides the starting point for a by now highly developed theory of locally convex $\tilde{\mathbb{C}}$-modules which to a large extent parallels the theory of locally convex vector spaces. Some of the main features of the theory are:

- The ups take over the role of seminorms.

- Completeness, metrizability, projective and inductive limits have been studied.

- There is a theory of barrelled and bornological $\tilde{\mathbb{C}}$-modules.

- Examples: $\mathcal{G}_{c}(\Omega)$ (corresponding to $\mathcal{D}(\Omega)$ ) is a strict inductive limit. $\mathcal{G}(\Omega)$ (corresponding to $\mathcal{C}^{\infty}(\Omega)$ ) is a Fréchet $\tilde{\mathbb{C}}$-module. The standard spaces $\mathcal{G}_{\tau}(\Omega), \mathcal{G}_{\mathcal{S}}(\Omega)$, $\mathcal{G}^{\infty}(\Omega)$, etc. can all be treated within the theory.

- Duality theory, study of

$$
\mathcal{L}(\mathcal{G}, \tilde{\mathbb{C}}):=\{T: \mathcal{G} \rightarrow \tilde{\mathbb{C}} \mid T \tilde{\mathbb{C}} \text {-linear and continuous }\}
$$


An example is the generalized delta distribution (point evaluation at $\tilde{x} \in \tilde{\Omega}): \delta_{\tilde{x}}=$ $u \mapsto u(\tilde{x}) \in \mathcal{L}\left(\mathcal{G}_{c}(\Omega), \tilde{\mathbb{C}}\right)$.

- Based on this, kernels of pseudodifferential operators can be constructed as elements of $\mathcal{L}\left(\mathcal{G}_{c}(\Omega \times \Omega), \tilde{\mathbb{C}}\right)$ (cf. also 6 ).

- Microlocal analysis in the dual of Colombeau algebras, see [1].

- A Hahn-Banach theorem is not attainable in general due to algebraic obstructions ([29]).

- Several open mapping and closed graph theorems and applications to $\mathcal{G}^{\infty}$-hypoellipticity are given in [12].

5. Conclusions and outlook. As can be seen from the above summary of results, Colombeau theory is currently undergoing a profound and far-reaching conceptual restructuring. Several branches of research that so far had been rather disconnected have seen fruitful and promising interactions. As a first example we have seen the strong links between global analysis and algebraic properties in Section 3. These will give rise to a new algebraic approach to non-smooth differential geometry. Moreover, the algebraic causality structures also mentioned in Section 3 are currently being pursued as a tool for generalizing the Hawking and Penrose singularity theorems of general relativity to space-times of low differentiability.

Interactions between algebra and PDE theory include topics like a refined study of hypoellipticity properties (which will require at least the rudiments of real algebraic geometry in the generalized setting). Moreover, as was indicated in Section 5, there are by now strong ties between functional analytic methods and the theory of pseudodifferential and Fourier integral operators. Similarly, there are close connections between such methods and variational problems of low regularity.

There already are examples of abstract (functional analytic) existence results for concrete analytical problems in PDE theory in the Colombeau framework, a direction of research which without doubt will gain importance in the near future. The hope here is to provide a toolkit (similar to the one available in classical analysis) of topological and algebraical methods for solving problems of non-smooth analysis and geometry.

Acknowledgments. This work was supported by projects P-16742 and Y-237 of the Austrian Science Fund.

\section{References}

[1] J. Aragona and S. O. Juriaans, Some structural properties of the topological ring of Colombeau's generalized numbers, Comm. Algebra 29 (2001), 2201-2230.

[2] J. Aragona, S. O. Juriaans, O. R. B. Oliveira and D. Scarpalézos, Algebraic and geometric theory of the topological ring of Colombeau generalized functions, Proc. Edinb. Math. Soc. 51 (2008), 545-564.

[3] H. A. Biagioni, A Nonlinear Theory of Generalized Functions, Lecture Notes in Mathematics 1421, Springer, Berlin, 1990.

[4] J. F. Colombeau, New Generalized Functions and Multiplication of Distributions, North Holland, Amsterdam, 1984. 
[5] J. F. Colombeau, Elementary Introduction to New Generalized Functions, North Holland, Amsterdam, 1985.

[6] A. Delcroix, Kernel theorems in spaces of tempered generalized functions, Math. Proc. Cambridge Philos. Soc. 142 (2007), 557-572.

[7] A. Delcroix, M. F. Hasler, S. Pilipović and V. Valmorin, Generalized function algebras as sequence space algebras, Proc. Amer. Math. Soc. 132 (2004), 2031-2038.

[8] G. de Rham, Differentiable Manifolds. Forms, Currents, Harmonic Forms, SpringerVerlag, Berlin, 1984.

[9] C. Garetto, Topological structures in Colombeau algebras: topological $\widetilde{\mathbb{C}}$-modules and duality theory, Acta Appl. Math. 88 (2005), 81-123.

[10] C. Garetto, Topological structures in Colombeau algebras: investigation of the duals of $\mathcal{G}_{c}(\Omega), \mathcal{G}(\Omega)$ and $\mathcal{G}_{\mathcal{S}}\left(\mathbb{R}^{n}\right)$, Monatsh. Math. 146 (2005), 203-226.

[11] C. Garetto, Microlocal analysis in the dual of a Colombeau algebra: generalized wave front sets and noncharacteristic regularity, New York J. Math. 12 (2006), 275-318.

[12] C. Garetto, Closed graph and open mapping theorems for topological $\widetilde{\mathbb{C}}$-modules and applications, Math. Nachr. 282 (2009), 1159-1188.

[13] J. Grant, E. Mayerhofer and R. Steinbauer, The wave equation on singular space-times, Commun. Math. Phys. 285 (2009), 399-420.

[14] M. Grosser, M. Kunzinger, M. Oberguggenberger and R. Steinbauer, Geometric Theory of Generalized Functions, Mathematics and its Applications 537, Kluwer Academic Publishers, Dordrecht, 2001.

[15] M. Kunzinger, Generalized functions valued in a smooth manifold, Monatsh. Math. 137 (2002), 31-49.

[16] M. Kunzinger and M. Oberguggenberger, Group analysis of differential equations and generalized functions SIAM J. Math. Anal. 31 (2000), 1192-1213.

[17] M. Kunzinger and R. Steinbauer, Generalized pseudo-Riemannian geometry, Trans. Amer. Math. Soc. 354 (2002), 4179-4199.

[18] M. Kunzinger, R. Steinbauer and J. A. Vickers, Intrinsic characterization of manifoldvalued generalized functions, Proc. London Math. Soc. 87 (2003), 451-470.

[19] M. Kunzinger, R. Steinbauer and J. A. Vickers, Generalised connections and curvature, Math. Proc. Cambridge Philos. Soc. 139 (2005), 497-521.

[20] M. Kunzinger, R. Steinbauer and J. A. Vickers, Sheaves of nonlinear generalized functions and manifold-valued distributions, Trans. Amer. Math. Soc. 361 (2009), 5177-5192.

[21] E. Mayerhofer, On Lorentz geometry in algebras of generalized functions, Proc. Roy. Soc. Edinb. 138 (2008), 843-871.

[22] M. Nedeljkov and S. Pilipović, Generalized function algebras and PDEs with singularities. A survey, Zb. Rad. (Beogr.) 11 (2006), 61-120.

[23] M. Oberguggenberger, Multiplication of Distributions and Applications to Partial Differential Equations, Pitman Research Notes in Mathematics 259, Longman, Harlow, 1992.

[24] M. Oberguggenberger and T. Todorov, An embedding of Schwartz distributions in the algebra of asymptotic functions, Internat. J. Math. Math. Sci.

[25] D. Scarpalézos, Colombeau's generalized functions: topological structures; microlocal properties. A simplified point of view. I, Bull. Cl. Sci. Math. Nat. Sci. Math. 25 (2000), 89-114.

[26] D. Scarpalézos, Colombeau's generalized functions: topological structures; microlocal properties. A simplified point of view. II, Publ. Inst. Math. (Beograd) (N.S.) 76 (90) (2004), $111-125$. 
[27] L. Schwartz, Sur l'impossibilité de la multiplication des distributions, C. R. Acad. Sci. Paris 239 (1954), 847-848.

[28] R. Steinbauer and J. A. Vickers, The use of generalized functions and distributions in general relativity, Classical Quantum Gravity 23 (2006), R91-R114.

[29] H. Vernaeve, Ideals in the ring of Colombeau generalized numbers, Comm. Algebra, to appear.

[30] H. Vernaeve, Isomorphisms of algebras of Colombeau generalized functions, Monatsh. Math., to appear. 